\title{
UHF PD Experiments on Real Power Transformer and its Frequency Characteristics Results
}

\author{
Hui Fan \\ State Grid Hebei \\ Electric Power \\ Research Institute \\ China \\ hbdysfh@126.com
}

\author{
Jialei $\mathrm{Hu}$ \\ Shanghai Jiao Tong \\ University, China \\ halleyhit@ \\ sjtu.edu.cn
}

\author{
Hongliang Liu \\ State Grid Hebei \\ Electric Power \\ Research Institute \\ China \\ hbdyylhl@163.com
}

\author{
Yi Yin \\ Shanghai Jiao Tong \\ University, China \\ yiny@ \\ sjtu.edu.cn
}

\author{
M. G. Danikas \\ Democritus \\ University of Thrace, \\ Greece \\ mdanikas@ \\ ee.duth.gr
}

\begin{abstract}
A number of methods have been used in partial discharge (PD) detection and recognition. Among these methods, ultra-high frequency (UHF) detection and recognition based on a single signal have attracted much attention. In this paper, a UHF PD detection system is built, and samples are acquired through experiments on a real power transformer. The received signal is decomposed into different frequency ranges through wavelet packet decomposition (WPD). In each frequency range, a pattern recognition neural network is built, and then the relationship between the information in that frequency range and PD type is described. By comparing the recognition accuracy of these networks, frequency range selection is optimized. In this specific case (the specific transformer, PD sources, and UHF sensors), results show that low frequency (156.25 $\mathrm{MHz}$ to $312.5 \mathrm{MHz})$ and high frequency ranges $(1093.75 \mathrm{MHz}$ to $1250 \mathrm{MHz})$ contain the most information for recognition. If a PD detection recognition system is to be designed, then the performance around these frequency ranges should be given attention.
\end{abstract}

Keywords- partial discharge; wavelet transform; neural network

\section{INTRODUCTION}

A power transformer is one of the most important equipment in electric power systems. The use of partial discharge (PD) measurement can help in the evaluation of the dielectric condition of high-voltage equipment. The electrical detection of PD can be regarded as an important tool for both, quality tests on HV equipment in the laboratory and diagnosis tests on site [1]. Recognition of PD types, as a part of PD measurement, contributes to PD source location and early fault diagnosis. For power transformers, PD is a symptom of insulation defect or degradation. Furthermore, different types of defects usually produce different PD waveforms, so it is possible to recognize the sources of the PD from its measured signal [2].

Using ultra-high frequency (UHF) sensor is an effective method to detect PD signals [3-7]. After receiving UHF PD signals, signal processing and analysis are supposed to reveal the type of potential insulation faults or defects, which helps to locate the PD source and determine needed follow-up maintenance. The traditional UHF PD recognition methods, represented by $n-q-\varphi$ pattern are based on statistical properties of PD phase distribution. Several algorithms for UHF PD recognition have been introduced. In 1991, neural network was introduced into this pattern [8].

To remove disturbance and improve recognition accuracy, many researchers have developed modified and novel methods. Among these new ideas, the two most attractive ones are the following: one idea is to introduce a filter as pretreatment, and the representative method is wavelet analysis. This improvement was first adopted in GIS [9], and then in power transformers [10-12]. The other idea is to extract information not from phase distribution but from a single PD signal. In [13], three features (skewness, kurtosis, and energy) were extracted. In [14], frequency characteristics of typical PD in GIS are studied, and their results serve as substantial criteria for optimal band width selection for high frequency detection.

The aforementioned methods are flexible, but more experiments are needed for validation, especially experiments in real power transformers rather than in tanks. In addition, in the use of wavelets, signals are decomposed into different frequency ranges, and a basic issue arises as to which frequency range contains more information. In this paper, a real power transformer with multiple PD sources is constructed. UHF method is employed to receive PD signals. Wavelet packet decomposition (WPD), being one of the most flexible methods that use wavelets is adopted to decompose PD signals into different frequency ranges. In each frequency range, a pattern recognition neural network is built, and then the relationship between information in a particular frequency range and PD type is described. By comparing the recognition accuracy of these networks, frequency range selection is optimized. Based on experimental results, frequency characteristics of UHF PD signals in power transformer are studied.

\section{ALGORITHM}

PD signal is a broadband signal, and its frequency range is generally between $300 \mathrm{MHz}$ and $1500 \mathrm{MHz}$ or even more. 
Furthermore, signals received by UHF sensors are usually polluted by different types of noise, which include continuous noise from communication systems, thermal noise from detection systems and periodic pulse-shaped noise from thyristor operations, among others. In this section, with the use of WPD, the received signal (composed of PD signal and noise) is decomposed into different frequency ranges. In each frequency range, a pattern recognition neural network is built. Obviously, a more accurate recognition means more useful information and less noise in that frequency range. Then, quantitative comparison is feasible.

\section{A. Wavelet Packet Decomposition}

Periodic signals are decomposed into different frequency ranges by Fourier transform, whereas nonperiodic signals are decomposed by WPD. WPD, which comes from discrete wavelet transform, is more flexible because it has more filters. Previously, discrete wavelet transform has been applied to UHF PD denoising $[15,16]$. In discrete wavelet transform, original signal $S$ is decomposed into $A_{1}$ and $D_{1} . A_{1}$ is the approximation component and contains a low-frequency component, whereas $D_{1}$ is a detail component containing the high-frequency component. Subsequently, $A_{1}$ is decomposed into $A A_{2}$ and $D A_{2}$ and is followed by $A A_{2}, A A A_{3}$ etc. After an $n$ level discrete wavelet transform, $n+1$ signals, containing components from different frequency ranges, are obtained. In WPD, after $S$ is decomposed into $A_{1}$ and $D_{1}, A_{1}$ is decomposed into $A A_{2}$ and $D A_{2}$, while $D_{1}$ is also decomposed. Highfrequency components are decomposed in WPD, but not in discrete wavelet transform.

For example, when decomposition level $n$ is set to 3 , signal $S$ is represented as

$$
S=D_{1}+D A_{2}+D A A_{3}+A A A_{3}
$$

in discrete wavelet transform, whereas its expansion is more flexible in WPD. For example, we can write

$$
S=D_{1}+D D A_{3}+A D A_{3}+A A_{2},
$$

and we can use other decomposition techniques depending on the situation.

In this paper, the PD signal is detected by a UHF sensor, and the sensor is linked to amplifier, and then oscilloscope. The sampling frequency of the original signal is $5 \mathrm{GHz}$, which is determined by the oscilloscope. However the working frequency range of the amplifier is $0 \mathrm{GHz}$ to $1.5 \mathrm{GHz}$, and that means the high frequency part is meaningless. To answer which frequency range contains more information, the original signal is decomposed into some different frequency ranges with the same width. The structure of the WPD used in this paper is shown in Figure 1. $D_{1}$ and $D A_{2}$ are high frequency part and are removed. $S_{1}$ to $S_{8}$ are 8 components of different ranges, and consequently, signal $S$ is represented as

$$
S=S_{1}+S_{2}+S_{3}+S_{4}+S_{5}+S_{6}+S_{7}+S_{8} .
$$

The frequency range of each component is shown in Table I.

In Figure 2, two signals, a point-to-plate type and a suspended-particle type (different PD types will be discussed in Section 3), are decomposed. Roughly, the two signals have different $S_{6}$ and $S_{8}$ components, which suggest that the frequency ranges of these two components are more important in PD recognition for this specific case. However, to be strict and precise, quantitative measures (discussed in Section 2.2) and numerous experiments (discussed in Section 3 ) are needed.

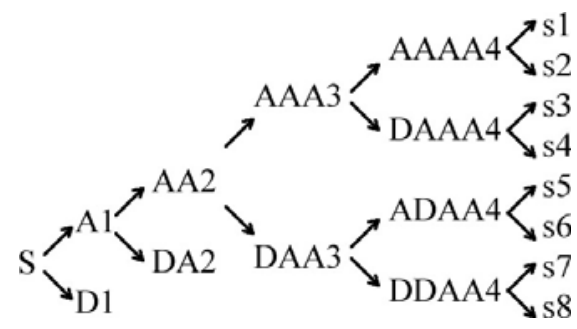

Fig. 1. Structure of WPD used in this paper

TABLE I. FREQUENCY RANGE OF EACH COMPONENT

\begin{tabular}{|c|c|}
\hline Component & Frequency Range (MHz) \\
\hline$S_{1}$ & 0 to 156.25 \\
\hline$S_{2}$ & 156.25 to 312.5 \\
\hline$S_{3}$ & 312.5 to 468.75 \\
\hline$S_{4}$ & 468.75 to 625 \\
\hline$S_{5}$ & 625 to 781.25 \\
\hline$S_{6}$ & 781.25 to 937.5 \\
\hline$S_{7}$ & 937.5 to 1093.75 \\
\hline
\end{tabular}

\section{B. Pattern Recognition Neural Networks}

The earliest neural networks probably date back to 1990 [17]. With the developments in computing science, neural networks are able to solve complex problems in the field of science. In this section, a neural network is used in PD recognition, and then the relationship between the information in a particular frequency range and PD type is described. Furthermore, to remove the effect of starting time (discussed in this section later), a pretreatment based on Fast Fourier Transform (FFT) is adopted. These two parts, neural networks and pretreatment, make up the special pattern recognition neural network in this paper.

\section{1) Neural Networks}

Essentially, neural networks are special functions designed to solve the problem of curve fitting. To neural networks, a PD signal is viewed as an input, whereas PD type is the output. Given enough samples, proper parameter settings, and training, the neural networks attempt to recognize PD type. If no neural network obtains high accuracy, then the recognition is an illposed problem, which suggests that the inputs do not have sufficient information. In other words, signals in that frequency range are less important.

A typical and basic neural network is shown in Figure 3; this is a two-layer feed-forward network with sigmoid hidden and softmax output neurons. In Figure $3, w$ is weight, $b$ is threshold, and $f$ and $r$ are activation functions. In this recognition neural network case, $f$ is sigmoid and $r$ is softmax. Outputs, namely, target vectors, have $N$ elements ( $N$ is the number of PD types); for each target vector, one element is 1 , and the others are 0 . In this network, inputs are to be classified into $N$ different PD types. The number of neurons in the hidden layer is an important parameter, as it affects the balance between precision and computational complexity. The number of neurons has been tried and determined by experience. 


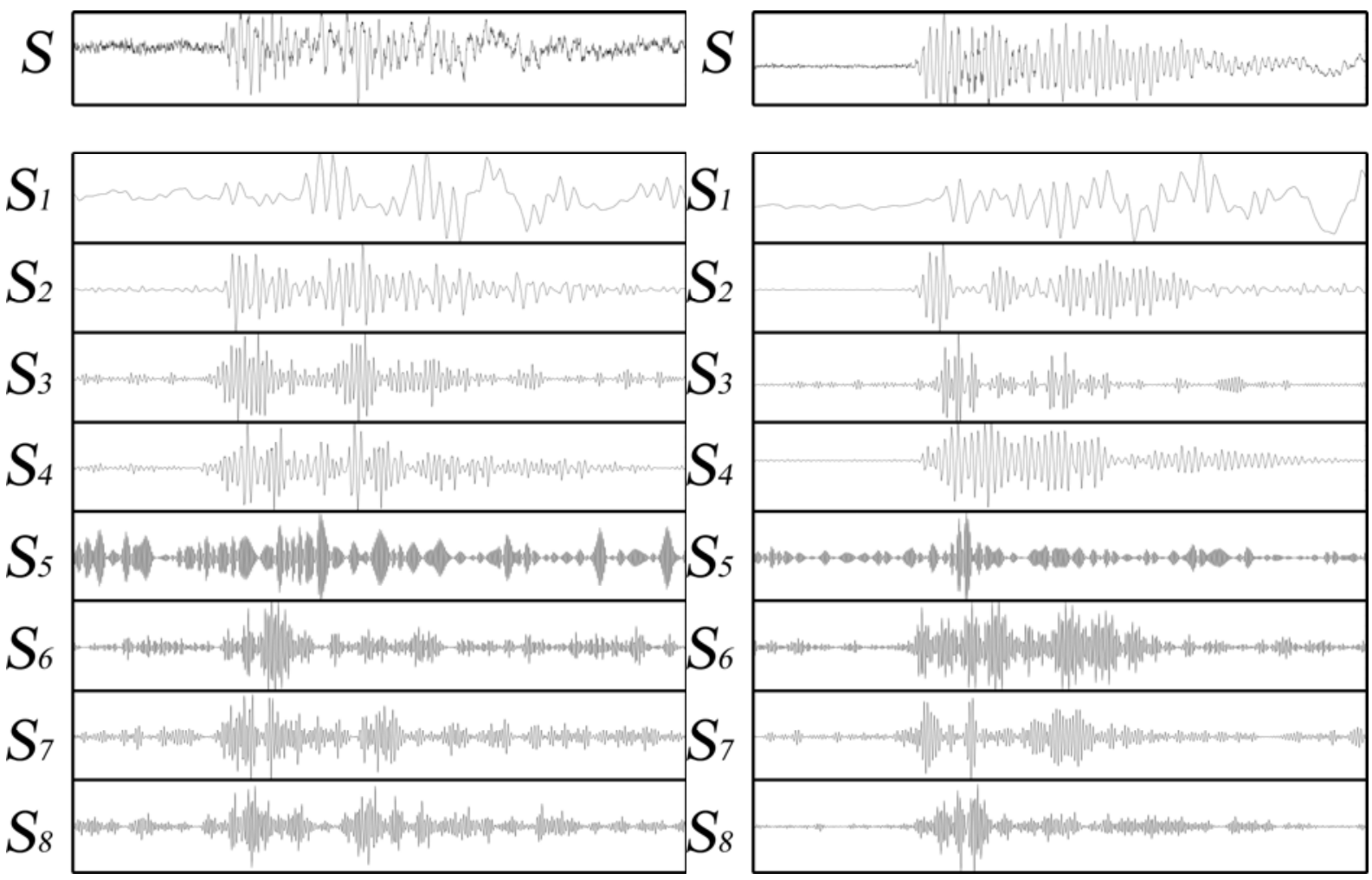

Fig. 2. Example of signal decomposition results

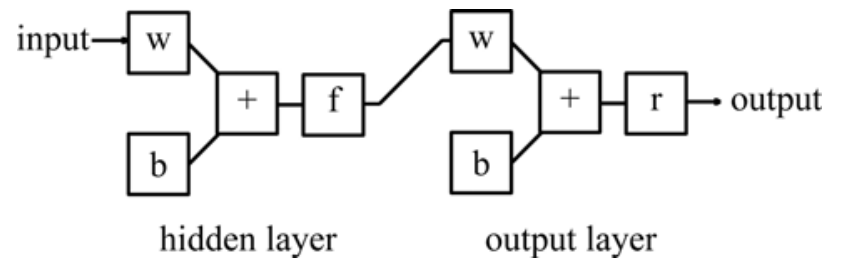

Fig. 3. Structure of a neural network.

\section{2) Pretreatment}

For neural networks, the input is a PD signal, which is a vector, namely, a time series,

$$
[S(-\mathrm{inf}), \ldots, S(-1), S(0), S(1), \ldots, S(\mathrm{inf})],
$$

where, $S(0)$ is around the start of PD. However, the input for a neural network is supposed to be a vector with a limited length,

$$
S S=[S(0), S(1), \ldots, S(l-1)],
$$

where, $l$ is the length. Obviously, $l$ should be large enough to contain sufficient information, but a more considerable length means a larger amount of calculation in recognition later. The length is drawn from experience and is suited to the exact conditions of measurement and the study purpose. In [18] and [19], some statistics suggest that PD duration may be several hundreds of nanoseconds. Furthermore, the precise location of $S(0)$ affects recognition accuracy to a great extent. Then, pretreatment is needed to remove the effect of starting time.

The current mature approach is the use of energy criterion [20]. The energy criterion is based on the observation that sound emission signals, in particular when of middle to bad quality, are predominantly characterised by a variation of their energy content, rather than a fluctuation of their frequency composition [21]. Suppose

$$
S=\left[S\left(-N_{1}\right), \ldots, S(-1), S(0), S(1), \ldots, S\left(N_{2}\right)\right]
$$

is a section of what we generate from the detection equipment. The energy curve $E(i)$ of the sampled signal $S(i)$ is defined here as the cumulative sum of amplitude values. The separation of the signal from the noise part and the realization of the criterion are managed with

$$
E E(i)=E(i)-i \delta=\sum_{k=-N_{1}}^{i}\left(S(k)^{2}\right)-i \delta, i=-N_{1}, \ldots, N_{2}
$$

where, a negative trend $\delta$ is introduced. The trend is dependent on the total energy $E\left(N_{2}\right)$ of the signal and the signal length. It is determined by

$$
\delta=\frac{E\left(N_{2}\right)}{N_{1}+N_{2}+1}
$$

The calculated partial energy curve $E E(i)$ features a global minimum, which is assumed to correspond with the signal arrival time or onset. Letting $S(0)$ be between $S\left(-N_{1}\right)$ and $S\left(N_{2}\right)$ is not difficult, given $N_{1}$ and $N_{2}$ are large enough. Then, we locate $S(0)$ after the minimum $E E$ is found.

Considering the sampling PD signal is discrete, when PD starting point is between two sampling points, an error is inevitable. Although the error is small and even smaller than the sampling period, it may be amplified in the subsequent steps. In the pretreatment, FFT is used to solve such a problem. 
FFT is widely applied in engineering, science, and mathematics [22]. FFT causes no information loss and requires only small computational cost. Another characteristic of FFT is that a change in amplitude characteristics in the frequency domain does not occur when a delay or advance occurs in the time domain. This characteristic means that the amplitude characteristics of PD signals do not change even when the precise location of $S(0)$ fails. Furthermore, experimental results (discussed in Section 4) suggest that amplitude characteristics contain sufficient information in PD recognition. The FFT is defined as

$$
\begin{gathered}
F(k)=\left|\sum_{i=0}^{l-1} S S(i) \omega^{i(k-1)}\right|, k=1,2, \ldots, l \\
\omega=e^{(-2 \pi j) / l}
\end{gathered}
$$

where, $i$ is the loop variable, $j$ is the imaginary unit, and || is the modulus of the complex number. Thus, $F$ is the amplitude characteristics of $S S$ and with the same length.

From the above neural networks and pretreatment, the algorithm, a special pattern recognition neural network is proposed, whose structure is shown in Figure 4. $S$ is the PD signal received by the UHF detection system. After applying the energy criterion, the PD starting time is roughly located. When the length $l$ is set, we have $S S$, a vector with a limited length. $F$ is the amplitude characteristics in the frequency domain, which are from the FFT of $S S$ and have the same length. At last, a neural network is trained. In the neural network, $F$ is the input, and PD type is the output. The effect of starting time is completely removed. Thus, the highest accuracy that the algorithm achieves is closely related to the information of the inputs. In addition, when $S_{i}$ takes the place of $S$, the relationship between information in each frequency range and PD type is measured.

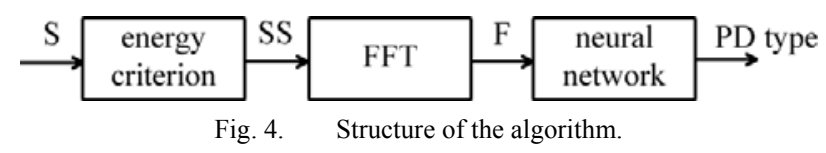

\section{EXPERIMENTS}

To acquire sufficient examples for the neural network training, full-scale experiments are conducted in a real power transformer with multiple PD sources. A UHF PD detection system is employed to receive PD signals. The system consists of the UHF sensor (UHF antenna), amplifier, oscilloscope, and computer. The power transformer is shown in Figure 5. Its rated power is $6300 / 6300 \mathrm{kVA}$, and its rated voltage is $110 / 35 / 10.5 \mathrm{kV}$. PD sources are placed inside the transformer and can be viewed through the windows. There are 9 measurement points on the transformer. Dismountable antenna can be installed on these points. The distance between source and antenna is $0.5 \sim 1.5 \mathrm{~m}$.

The PD sources have five types, namely, point to plate (a sharp protrusion on an electrode), surface (slender) discharge (surface contamination on an insulating support), surface (flat) discharge (surface contamination on another insulating support), suspended particle (a loose metallic particle in transformer oil), and cavity discharge (a cavity within solid insulation). When we apply a voltage on these PD sources, PD signals are generated, and the signals vary with the voltage. The media between PD source and measurement point is oil. The transformer and PD sources have been used in similar experiments $[11,23]$.

In the UHF PD detection system, the UHF sensor is the most important part. Three types of UHF sensor, namely, Goubau antenna, monopole antenna, and discone antenna (Figure 6), have been tested. The design requirements of all three types are the same. For each UHF sensor type and each PD type, some samples are acquired through experiments under different voltages. AC voltage is applied to the PD source. With the voltage increasing stepwise, the detection system begins to receive PD signals. When the voltage is too high such that the noise suddenly increases or the waveform is obviously polluted and meaningless, the voltage is removed. The PD sources are different. Thus, the numbers of samples across groups are not the same. Details are shown in Table II.

A total of 9970 examples are obtained. Each sample has a single PD signal. These samples are divided into three groups by UHF sensor type. In each group, the PD signals are decomposed by WPD into eight components with different frequency ranges. Furthermore, five wavelets, namely, Haar, $\mathrm{db} 2, \mathrm{db} 8$, sym2, and sym8, are tested. "Haar" is the most basic wavelet. "db" refers to Daubechies wavelets, and "sym" refers to symlets. In each component, the special pattern recognition neural network is trained. Three sensor types, eight frequency ranges, and five wavelet types result in $120(3 \times 8 \times 5)$ neural networks in all. By comparing the recognition accuracy of these networks, sensor type, frequency range, and wavelet type selection is optimized.During pretreatment, length $l$ is 1000 . As the sampling frequency is $5 \mathrm{GHz}$, the first $200 \mathrm{~ns}$ of PD signals from the starting point, which is enough for recognition based on our experience, are saved. The number of neurons in the hidden layer is set to 50 in neural networks. As results show, such complexity is enough to solve this problem. These neural networks are trained with scaled conjugate gradient backpropagation. Sample division during training is $70 \%$ training, $15 \%$ validation, and $15 \%$ testing.

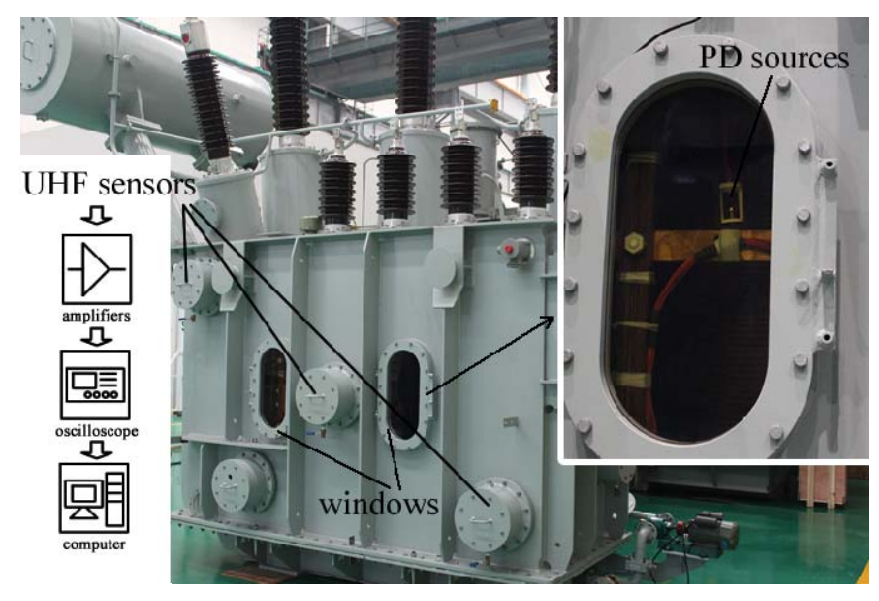

Fig. 5. Power transformer used in the experiments. 


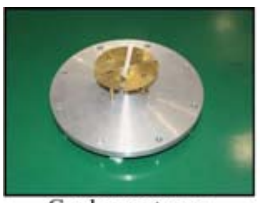

Goubau antenna

Fig. 6.

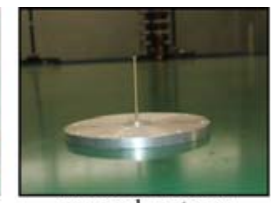

monopole antenna

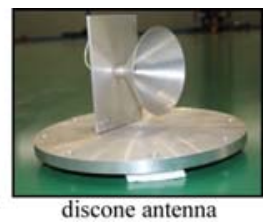

discone antenna
UHF sensors used in experiments.

TABLE II. SAMPLE DETAILS

\begin{tabular}{|c|c|c|c|}
\hline Sample Number & Goubau & monopole & discone \\
\hline point to plate & 281 & 859 & 1718 \\
\hline surface (slender) & 360 & 360 & 720 \\
\hline surface (flat) & 551 & 551 & 1102 \\
\hline suspended particle & 566 & 566 & 1132 \\
\hline cavity & 301 & 301 & 602 \\
\hline
\end{tabular}

IV. RESULTS

\section{A. Single Component}

In the case of Haar wavelet and Goubau antenna, for example, samples in the Goubau group are decomposed into $S_{1}$ to $S_{8}$. In each $S_{i}$, a special pattern recognition neural network is trained. The recognition accuracies of the different components are listed in Table III. The best accuracy is $S_{2}$ and the worst is $S_{5}$. That means, $S_{2}$ contains the highest amount of useful information, whereas $S_{5}$ contains the least amount of useful information. In addition, recognition accuracy is quite high, which suggests that amplitude characteristics contain enough information for PD recognition in this specific case, as mentioned in the previous section. Results from other wavelets and other antenna types are shown in Figure 7. Comparison of the results by wavelet shows that in the Goubau and monopole groups, wavelet types only slightly influence recognition accuracy, which does not hold true in the discone group. A comparison between the results by antenna type shows that the first two groups are better than the last one. Thus, the use of discone antenna is not recommended for this specific case.

More notably, when the 15 subfigures in Figure 7 are added together, we have the distribution characteristic of a single component, as shown in Figure 8. A histogram bar is used to plot the quantitative distribution. Elements of recognition accuracy in all the settings (wavelets and antenna types) are sorted into five equally spaced bins along the $x$-axis between the minimum and maximum values. Bins are displayed as rectangles such that the height of each rectangle indicates the number of elements in the bin. The recognition accuracy of $S_{5}$ is clearly located mainly around 0.65 , whereas that of $S_{2}$ is located around 0.9. Thus, the $S_{5}$ frequency component (from $625 \mathrm{MHz}$ to $781.25 \mathrm{MHz}$ ) contains less information for PD recognition. It should be noted that the distribution characteristic reflects the overall performance of different frequency ranges.

\section{B. Multi-adjacent Components}

In a similar way, recognition accuracies of $S_{1}+S_{2}$ and $S_{1}+S_{2}+S_{3}$ are compared. Results are shown in Figure 9. Bars in warm color are more likely to be in low accuracy ranges. An obvious trend is that the low-frequency range contains the most information, whereas the mid-frequency range contains the least information.

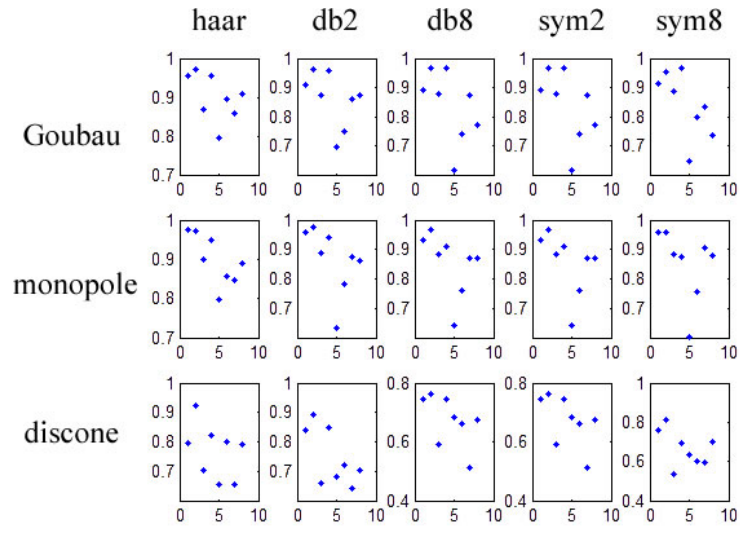

Fig. 7. Results with different wavelets and antenna types.

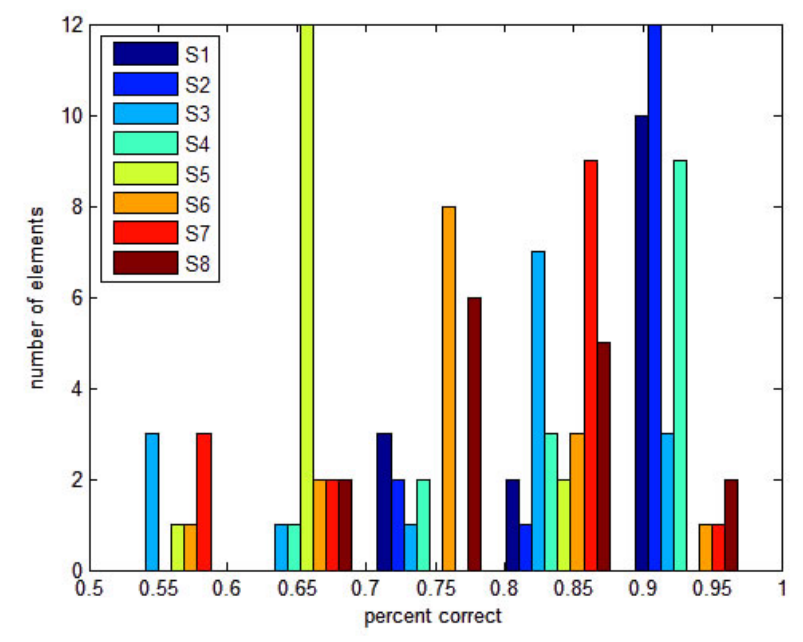

Fig. 8. Distribution of single component.

TABLE III. RECOGNITION ACCURACIES IN HAAR AND GOUBAU CASE

\begin{tabular}{|c|c|}
\hline Component & Recognition Accuracy \\
\hline$S_{1}$ & 0.956289461 \\
\hline$S_{2}$ & 0.972316659 \\
\hline$S_{3}$ & 0.869354055 \\
\hline$S_{4}$ & 0.954346770 \\
\hline$S_{5}$ & 0.796017484 \\
\hline$S_{6}$ & 0.895094706 \\
\hline$S_{7}$ & 0.856726566 \\
\hline$S_{8}$ & 0.907236523 \\
\hline
\end{tabular}

\section{Quantitative Results}

Although a figure is an intuitional representation of results, a quantitative study is necessary to generate precise results. Mean value and variance are basic statistics. The statistical properties of Figures 8 and 9 are listed in Table IV. A high mean value means good performance, and a low variance 
indicates stability. Compared with the single-component group, the multi-adjacent-component groups have a better performance (higher average and lower deviation). The results suggest that even if some components, $S_{5}$ for example, contain the least useful information, they still facilitate recognition. The low components contain the most information, especially $S_{2}$ (from $156.25 \mathrm{MHz}$ to $312.5 \mathrm{MHz}$ ); the mid-components contain the least information, especially $S_{5}$ (from $625 \mathrm{MHz}$ to $781.25 \mathrm{MHz}$ ). In addition, components in the high-frequency range, $S_{8}(1093.75 \mathrm{MHz}$ to $1250 \mathrm{MHz})$ for example, are also important in recognition. However, we need to stress that the results pertain to a specific case. To verify the universality of the results, additional experiments and analysis are required.
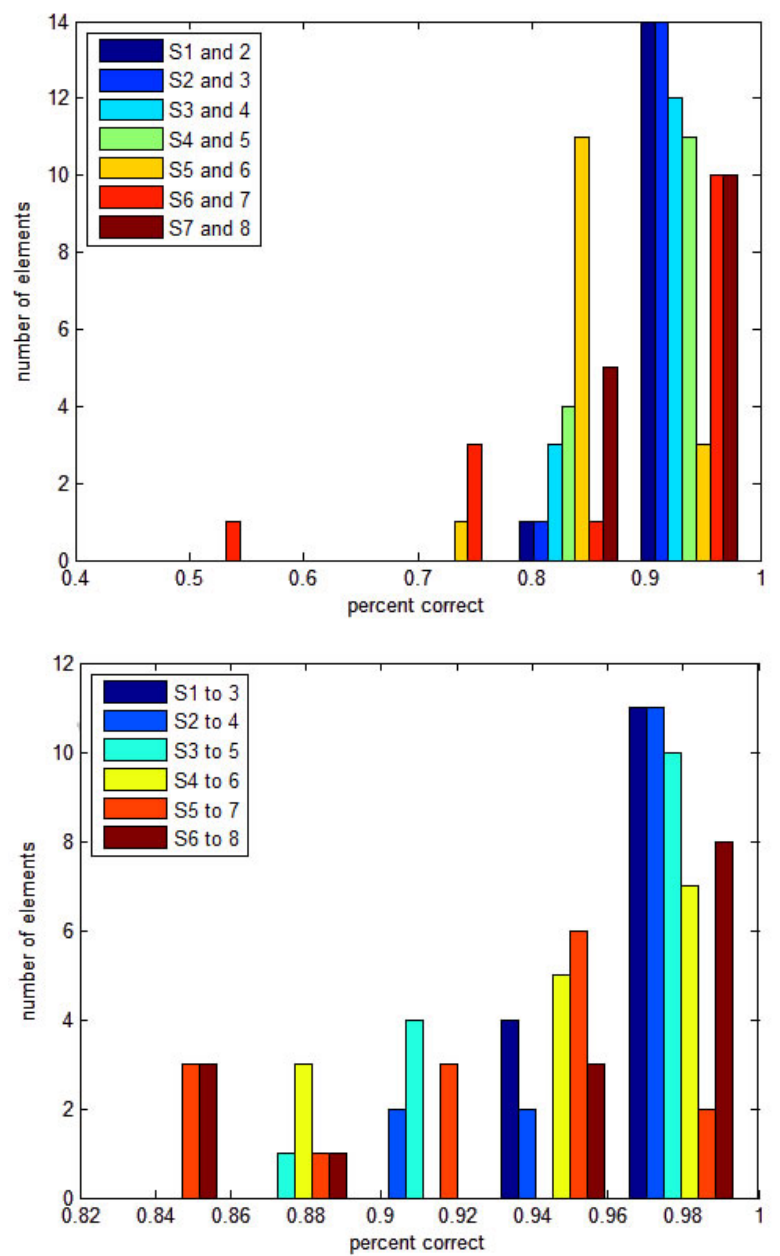

Fig. 9. Distribution of multi-adjacent components.

\section{DISCUSSION}

One of the most fundamental and important mission for the UHF PD detection is to tell the PD type. To improve the recognition accuracy, a number of advanced algorithms and after-treatments are introduced. However, a basic question is whether we obtain enough information by the detection system. In this paper, quantitative results for this specific case (the specific transformer, PD sources, and UHF sensors) show that the low-frequency range $(156.25 \mathrm{MHz}$ to $312.5 \mathrm{MHz})$ and high-frequency range (1093.75 MHz to $1250 \mathrm{MHz}$ ) contain the most information for recognition.

TABLE IV. QUANTITATIVE RESULtS

\begin{tabular}{|c|c|c|}
\hline Component(s) & Average & Standard Deviation \\
\hline$S_{1}$ & 0.8803 & 0.0068 \\
\hline$S_{2}$ & 0.9215 & 0.0060 \\
\hline$S_{3}$ & 0.7929 & 0.0183 \\
\hline$S_{4}$ & 0.8827 & 0.0087 \\
\hline$S_{5}$ & 0.6689 & 0.0035 \\
\hline$S_{6}$ & 0.7531 & 0.0056 \\
\hline$S_{7}$ & 0.7724 & 0.0207 \\
\hline$S_{8}$ & 0.7976 & 0.0073 \\
\hline \hline$S_{1}+S_{2}$ & 0.9591 & 0.0011 \\
\hline$S_{2}+S_{3}$ & 0.9548 & 0.0018 \\
\hline$S_{3}+S_{4}$ & 0.9439 & 0.0025 \\
\hline$S_{4}+S_{5}$ & 0.9223 & 0.0035 \\
\hline$S_{5}+S_{6}$ & 0.8430 & 0.0025 \\
\hline$S_{6}+S_{7}$ & 0.8511 & 0.0188 \\
\hline$S_{7}+S_{8}$ & 0.9093 & 0.0052 \\
\hline \hline$S_{1}+S_{2}+S_{3}$ & 0.9702 & 0.0004 \\
\hline$S_{2}+S_{3}+S_{4}$ & 0.9726 & 0.0007 \\
\hline$S_{3}+S_{4}+S_{5}$ & 0.9586 & 0.0014 \\
\hline$S_{4}+S_{5}+S_{6}$ & 0.9504 & 0.0014 \\
\hline$S_{5}+S_{6}+S_{7}$ & 0.9191 & 0.0018 \\
\hline$S_{6}+S_{7}+S_{8}$ & 0.9362 & 0.0029 \\
\hline & & \\
\hline
\end{tabular}

The basic key conclusions for different frequency ranges are listed as follows:

- Regarding the very low frequency range (lower than 156.25 MHz), it has to be pointed out that it is not the best range for PD recognition. This may be partly because the signal pollution by radio broadcasting, whose frequency in China is about $100 \mathrm{MHz}$.

- $\quad$ Regarding the low frequency range (156.25 MHz to 312.5 $\mathrm{MHz}$ ) it has to be pointed out that it is the best range for recognition. However, if the sensor involves such range only, it may be a severe problem to distinguish between $\mathrm{PD}$ and corona. The middle low range is effective but not practical.

- $\quad$ Regarding the middle frequency range $(312.5 \mathrm{MHz}$ to $1093.75 \mathrm{MHz}$ ) it has to be pointed out that it is shown to be the worst range. That suggest the main pollution is in this range, especially the mobile phone signal whose range is about $1000 \mathrm{MHz}$ in China. This pollution is strong and is impossible to be shielded.

- $\quad$ Regarding the high frequency range (1093.75 $\mathrm{MHz}$ to $1250 \mathrm{MHz}$ ) it has to be pointed out that it found to be near the best and common UHF system emphasizes it. Also, it is the key to distinguish between PD and corona.

- Regarding the very high frequency range (higher than $1250 \mathrm{MHz}$ ) it has to be pointed out that it almost exceeds the effective range of the detecting system. It is really hard 
to increase the working frequency range of the system, on e.g. antenna design and amplifier bandwidth design.

Furthermore, comparisons of the method proposed in the present paper should be made with recent work [24], where four classic PD patterns, including floating PD, needle PD, oil learance $\mathrm{PD}$ and void $\mathrm{PD}$ in press-board were studied. Moreover, it would be useful if the proposed technique here can be tried and studied in PD detection with different detection methods [25].

\section{CONCLUSIONS AND PROSPECTS}

In this paper, the UHF method is employed to receive PD signals. The received signal is decomposed into different frequency ranges by using WPD. In each frequency range, a pattern recognition neural network is built, and then the relationship between the information in a specific frequency range and PD type is described. By comparing the recognition accuracy of these networks and through experiments, information in different frequency ranges is studied. Quantitative results for this specific case (the specific transformer, PD sources, and UHF sensors) show that the lowfrequency range $(156.25 \mathrm{MHz}$ to $312.5 \mathrm{MHz})$ and highfrequency range $(1093.75 \mathrm{MHz}$ to $1250 \mathrm{MHz})$ contain the most information for recognition. If a PD detection system is to be designed, then the performance around these frequency ranges should be given special attention. Furthermore, we can recognize PD type based on a single signal, and several frequency ranges are important. However, we stress again that all these results are applicable to a specific case only. Additional experiments are necessary. Moreover, a theoretical explanation may be provided in future studies.

\section{REFERENCES}

[1] E. Lemke, P. Schmiegel, Introduction to fundamentals of PD diagnostics, Lemke Diagnostics GmbH, Germany, 2000

[2] R. Bartnikas, "Partial discharges. Their mechanism, detection and measurement", IEEE Transactions on Dielectrics and Electrical Insulation, Vol. 9, No. 5, pp. 763-808, 2002

[3] B. F. Hampton, R. J. Meats, "Diagnostic measurements at UHF in gas insulated substations", IEE Proceedings C on Generation, Transmission and Distribution, Vol. 135. No. 2, pp. 137-145, 1988

[4] J. Pearson, J., B. F. Hampton, M. D. Judd, B. Pryor, P. F. Coventry, "Experience with advanced in-service condition monitoring techniques for GIS and transformers", IEE Colloquium on HV Measurement, Condition Monitoring and Associated Database Handling Strategies, London, UK, 3 June 1998, pp. 8-8, 1998

[5] K. Raja, T. Floribert, "Comparative investigations on UHF and acoustic PD detection sensitivity in transformers", Conference Record of the 2002 IEEE International Symposium, Boston, MA, USA, pp. 150-153, 7-10 April, 2002

[6] M. D. Judd, L. Yang, I. B. B. Hunter, "Partial discharge monitoring of power transformers using UHF sensors. Part 2: Field Experience", IEEE Electrical Insulation Magazine, Vol. 21, No. 3, pp. 5-13, 2005

[7] H. R. Mirzaei, A. Akbari, E. Gockenbach, K. Miralikhani, "Advancing new techniques for UHF PD detection and localization in the power transformers in the factory tests", IEEE Transactions on Dielectrics and Electrical Insulation, Vol. 22, No. 1, pp. 448-455, 2015

[8] H. Suzuki, T. Endoh, "Pattern recognition of partial discharge in XLPE cables using a neural network", Proceedings of the $3^{\text {rd }}$ International Conference on Properties and Applications of Dielectric Materials, Tokyo, Japan, pp. 43-48, 8-12 July, 1991
[9] Z. M. Li, Y. P. Feng, J. Q. Chen, X. Z. Zhang, "UHF partial discharge monitoring in GIS and signal processing", Proceedings of 1998 International Symposium on.Electrical Insulating Materials, Toyohashi, Japan, pp. 67-70, 27-30 September, 1998

[10] L. Yang, M. D. Judd, "Recognising multiple partial discharge sources in power transformers by wavelet analysis of UHF signals", IEE Proceedings-Science, Measurement and Technology, Vol. 150, No. 3 pp. 119-127, 2003

[11] Y. Wang, J. Wu, Z. Li, Y. Yin, "Research on a practical de-noising method and the characterization of partial discharge UHF signals", IEEE Transactions on Dielectrics and Electrical Insulation, Vol. 21, No. 5, pp. 2206-2216, 2014

[12] K. Wang, J. Li, S. Zhang, R. Liao, "A hybrid algorithm based on s transform and affinity propagation clustering for separation of two simultaneously artificial partial discharge sources", IEEE Transactions on Dielectrics and Electrical Insulation, Vol. 22, No. 2, pp. 1042-1060, 2015

[13] H. H. Sinaga, B. T. Phung, T. R. Blackburn, "Recognition of single and multiple partial discharge sources in transformers based on ultra-high frequency signals", IET Generation, Transmission \& Distribution Vol. 8, No. 1, pp. 160-169, 2014

[14] W. Gao, D. Zhao, D. Ding, S. Yao, "Investigation of frequency characteristics of typical PD and the propagation properties in GIS", IEEE Transactions on Dielectrics and Electrical Insulation, Vol. 22, No. 3, pp. 1654-1662, 2015

[15] L. Yang, M. D. Judd, C. J. Bennoch, "Denoising UHF signal for PD detection in transformers based on wavelet technique", Annual report on Electrical Insulation and Dielectric Phenomena (CEIDP), pp. 166-169, 17-20 October, 2004

[16] S. Sagar, J.Raghunath, J. Amarnath, S. Narasimham, "Wavelet Transform Technique for Denoising of UHF PD Signals in GIS", Third International Conference on Industrial and Information Systems (ICIIS 2008), pp. 1-4, Kharagpur, India, 8-10 December, 2008

[17] S. McCulloch, S. Warren, W. Pitts, "A logical calculus of the ideas immanent in nervous activity", The Bulletin of Mathematical Biophysics, Vol. 5, No. 4, pp. 115-133, 1943

[18] I. Portugues, P. J. Moore, I. A. Glover, "The effect of multipath in time domain characterization of partial discharges", Proceedings of the 7th International Conference on. Properties and Applications of Dielectric Materials, Vol. 1, pp. 311-314, 2003

[19] Z. Zheng, K. Tan, "Comparison of feature extraction methods in partial discharge waveform recognition", Annual Report Conference on Electrical Insulation and Dielectric Phenomena, USA, 14-17 October, 2001

[20] S. Markalous, S. Tenbohlen, K. Feser, "Detection and Location of Partial Discharges in Power Transformers using acoustic and electromagnetic signals", IEEE Transactions on Dielectrics and Electrical Insulation, Vol. 15, No. 6, pp. 1576-1583, 2008

[21] C. U. Grosse, H. Reinhardt, "Schallemissionsquellen automatisch lokalisieren: Entwicklung eines Algorithmus", Materialprüfung, Vol. 41, No. 9, pp. 342-347, 1999

[22] J. W. Cooley, J. W. Tukey, "An algorithm for the machine calculation of complex Fourier series", Mathematics of Computation, Vol. 19, No. 90, pp. 297-301, 1965

[23] Y. Wang, C. Zhu, Q. Wang, Z. Wang, Y. Yin, "Processing of partial discharge ultra-high frequency signals from a true size transformer", 2013 IEEE International Conference on Solid Dielectrics (ICSD), Bologna, Italy, pp. 1012-1015, 30 June - 4 July 2013

[24] X. Zhao, Y. H. Cheng, Y. -P. Meng, M. G. Danikas, "Applying WPD and SVD to classification of EM wave induced by partial discharge in power transformer", Journal of Electrical Engineering, Vol. 64, No. 4, pp. 222-229, 2013

[25] R. Sarathi, P. D. Singh, M. G. Danikas, "Characterization of partial discharges in transformer oil insulation under $\mathrm{AC}$ and DC voltage using Acoustic Emission Technique”, Journal of Electrical Engineering, Vol. 58, No. 2, pp. 91-97, 2007 